ARTÍCULOS

\title{
La formación investigativa en función del emprendimiento social en la Universidad actual
}

\section{Research training based on social entrepreneurship in the current University}

\author{
Breijo Worosz, Taymi
}

Taymi Breijo Worosz

taimi.breijo@upr.edu.cu

Universidad de Pinar del Río "Hermanos Saíz", Cuba

\section{Delectus}

Instituto Nacional de Investigación y Capacitación Continua, Perú ISSN-e: 2663-1148

Periodicidad: Semestral

vol. 2, núm. 2, 2019

publicaciones.iniccperu@gmail.com

Recepción: 11 Julio 2019

Aprobación: 15 Diciembre 2019

Publicación: 28 Diciembre 2019

URL: http://portal.amelica.org/ameli/journal/390/3902683004/

Esta licencia permite a otros entremezclar, ajustar y construir a partir de su obra con fines no comerciales, y aunque en sus nuevas creaciones deban reconocerle su autoría y no puedan ser utilizadas de manera comercial, no tienen que estar bajo una licencia con los mismos términos.

\section{(c) (i) (9)}

Esta obra está bajo una Licencia Creative Commons AtribuciónNoComercial 4.0 Internacional.
Resumen: El problema científico que plantea esta investigación es icómo perfeccionar el proceso de formación investigativa en función del emprendimiento social en la universidad actual? Como objetivos específicos se pretende fundamentar, desde el punto de vista pedagógico y didáctico, el proceso de formación investigativa de los profesionales en función del emprendimiento en la universidad actual. Para ello se utilizó una metodología basada en el paradigma cualitativo, que pondera la interpretación de los resultados desde una concepción dialéctica. Se aporta una estrategia pedagógica, encaminada a elevar de forma progresiva la innovación, creatividad y el espíritu emprendedor de los estudiantes universitarios, al apropiarse de competencias investigativas que les permiten dar solución a problemas del ejercicio de la profesión, desde la gestión del docente universitario.

Palabras clave: formación investigativa, emprendimiento, gestión del docente.

Abstract: The scientific problem posed by this research is how to improve the process of research training based on social entrepreneurship in the current university. As specific objectives, it is intended to base from the pedagogical and didactic point of view, the process of research training of professionals based on entrepreneurship in the current university. For this, a methodology based on the qualitative paradigm was used, which ponders the interpretation of the results from a dialectical conception. A pedagogical strategy is provided, aimed at progressively raising the innovation, creativity and entrepreneurial spirit of university students by appropriating research skills that allow them to solve problems in the practice of the profession from the management of the university teacher.

Keywords: Research training, entrepreneurship, university teacher management.

\section{INTRODUCCIÓN}

Dentro del escenario regional se han definido, tras los debates realizados en diversos eventos, las tendencias innovadoras de la educación superior, la que, según la UNESCO, se enfrenta a un proceso de reorganización 
y flexibilización de sus estructuras académicas y al fomento de la interdisciplinariedad. De este modo se evidencian esfuerzos por lograr: acciones estratégicas en el actual proceso de cambios educativos en los procesos que en ella se gestionan, y que ubican a la universidad en una función clave, en los programas de formación de los profesionales.

La práctica social ha demostrado en el decurso histórico de la humanidad, que la Universidad es la institución social que tiene como función la de conservar y desarrollar la cultura de la sociedad, mediante sus procesos sustantivos. Estos procesos universitarios se convierten en la vía más eficiente y sistemática que la sociedad contemporánea escoge para la conservación y desarrollo de su cultura.

La investigación en la universidad contemporánea, adquiere la dimensión de agente dinamizador de los procesos universitarios, dirigido a impulsar la formación de profesionales capaces de enfrentar y resolver los desafíos de una sociedad cada vez más compleja por el continuo desarrollo tecnológico.

Para ello emerge en la actualidad con mucha presencia, la necesidad de la formación de profesionales emprendedores, que estén preparados para dar respuestas innovadoras y creativas a los problemas sociales.

La educación superior cubana, inmersa en las nuevas transformaciones educacionales, sustenta la necesaria integración entre lo académico, lo laboral y lo investigativo como eje conductor del proceso para el tránsito hacia la excelencia universitaria en la formación profesional.

Los grandes retos a los que se enfrenta nuestra sociedad para consolidar sus logros y mantener sus aspiraciones, requieren de profesionales capaces y comprometidos con nuestro proyecto social. Esta investigación se inserta en la línea de investigación de la Universidad de Pinar del Rio: "Gestión integral del proceso de formación de profesionales”, que desde la gestión de la autora principal de este trabajo se coordina desde el Centro de Estudios en Ciencias de la Educación de esta institución.

Para ello se realizó un análisis de los cambios generados en la formación de investigadores desde el nuevo modelo de formación del profesional en Cuba, que emergen como plataforma de acción teórico-práctica.

La investigación estuvo dirigida al estudio de los marcos teóricos, conceptuales e históricos para la determinación de las características esenciales del proceso de formación investigativa en función del emprendimiento en el contexto internacional y cubano y a la determinación de las principales manifestaciones en la Universidad de Pinar del Río.

Así se reconoce como problema científico a resolver: ¿Cómo perfeccionar el proceso de formación investigativa en función del emprendimiento social desde la gestión del docente universitario en la Universidad de Pinar del Río?

Las ideas expuestas resumen en esencia, el interés por realizar la presente investigación. Su objetivo está dirigido a: Fundamentar desde el punto de vista pedagógico y didáctico, el proceso de formación investigativa de los profesionales en función del emprendimiento desde el perfeccionamiento de la gestión del docente universitario.

Para el logro de lo anterior se emplearon los siguientes métodos de Investigación, que par- ten de asumir como método general, el método dialéctico, en tanto posibilitó: el estudio del objeto como un proceso y la fundamentación e integración de los otros métodos utilizados.

\section{DESARROLLO}

\section{La formación investigativa en el contexto internacional. Principales características}

El escenario actual, complejo y globalizado exige de una universidad donde se privilegie la investigación, como proceso consciente que se desarrolla en el marco universitario, desde donde el futuro profesional debe asimilar lo anterior y lograr cada vez, mayores niveles de integración a la realidad objetiva. El proceso de investigación científica tiene que responder a la necesidad de incrementar los niveles de vida espirituales y materiales de la sociedad. 
$\mathrm{Al}$ respecto, el Documento de Política para el Cambio y el Desarrollo en la Educación Superior reconoce que la nueva universidad debe convertirse en una comunidad dedicada plenamente a la investigación, la creación y la difusión del conocimiento, al progreso de la ciencia, y que participe en el desarrollo de innovaciones e invenciones tecnológicas (Unesco, 1995, p. 3).

Así emerge una dialéctica entre investigación y profesión, encaminada a la formación de profesionales capaces de transformar su realidad y por ende desarrollarla. El desconocer lo científico-investigativo, convierte a la universidad en obsoleta e ineficiente, el desconocer lo profesional, la aleja de la vida y pierde su vínculo con la realidad (Álvarez y Sierra, 1997, p. 21).

De tal forma el proceso de investigación se convierte en hilo conductor de las necesarias transformaciones en la educación superior. La adquisición de conocimientos y la capacidad para aplicarlos y la aprehensión y puesta en práctica de los procesos tecnológicos se reconocen, hoy en día, como las principales fuentes de valor agregado. Comprender y gestionar la actividad científica como un componente esencial de la calidad, pertinencia y viabilidad de la universidad, es el primer paso para garantizar la pertinencia de los procesos universitarios.

En la actualidad se evidencia que las universidades desarrollan sus investigaciones en a partir de las demandas dependencia de los intereses de las grandes empresas, lo que afecta al desarrollo de investigaciones básicas dirigidas a resolver los problemas de la sociedad en su conjunto. El proceso investigativo se ve limitado, en la mayoría de los casos, por necesidades financieras que solo pueden ser resueltas por grandes compañías que financian las investigaciones en interés privado.

En consecuencia, la producción del conocimiento y las capacidades de investigación se concentran en los países desarrollados, lo que unido a la ampliación del proceso de transferencia del conocimiento de las universidades hacia las entidades económicas privadas, tiende a convertir a la universidad en una institución cada vez más funcionalista y desnaturalizada de su esencia (Munárriz, 2008, p. 2).

Un estudio exploratorio realizado sobre el proceso de formación investigativa a los centros de educación superior permite identificar los siguientes elementos que emergen como regularidades (Alfonso, 2002, p. 2):

- La no integración con la realidad social.

- La escasa relación con el perfil profesional y con los problemas propios de la profesión.

- La no vinculación con los objetivos específicos y generales de las carreras.

- La insuficiente relación con los principios y categorías de la ciencia

- Su no utilización como recurso didáctico para el aprendizaje.

- Orientada principalmente a su aplicación comercial.

\section{La formación de habilidades investigativas en el contexto cubano. Principales características}

En 1728 con la creación de La Real Pontificia Universidad de San Jerónimo se da inicio a los estudios universitarios en Cuba. Los primeros programas universitarios se caracterizaron por un profundo contenido escolástico y aristotélico en su visión más reaccionaria. El estudio directo de la naturaleza, la experimentación, la actitud intelectual desembarazada de dogmas tardaron en aparecer (Vitier, 2002, p. 17).

En la segunda mitad del propio siglo, 1773, se crea El Real Colegio de San Carlos y San Ambrosio. Figuras insignes de la cultura cubana, Agustín Caballero, Varela, Luz y Caballero, Saco, entre otros, inspirados por la necesidad de reformar los estudios universitarios, los preceptos iluministas y las ideas avanzadas de la Constitución de Cádiz (1820), le alentaron aires de modernidad y, en cierto modo, de cientificidad, lo que explica que superase en el ámbito académico a la propia universidad.

Durante toda la etapa colonial, los estudios universitarios estuvieron marcados por la influencia absoluta de la perspectiva filosófica y académica del escolasticismo.

En la primera mitad del siglo XIX se desarrollan un grupo de propuestas de reformas dirigidas a desterrar de los estudios universitarios el dogmatismo y el normativismo imperante. La propuesta de reforma ofrecida por Enrique José Varona es muestra de ello. Los fundamentos de su modelo fueron: 
- Desarrollar una enseñanza objetiva y científica.

- Desarrollar métodos basados en la experimentación.

- Formar profesores que enseñaran como se aprendía, como se estudiaba y como se investigaba.

Se convirtió en el centro de muchas polémicas, la necesidad de desarrollar la investigación científica en los estudios universitarios. El Congreso Internacional de Universidades, celebrado en La Habana en 1930, tuvo como uno de los temas centrales la investigación universitaria. Se proponen: primero, que los estudiantes se incorporen, como investigadores asociados, a las investigaciones desarrolladas por los profesores en sus cátedras y segundo, implementar las tesis de doctorado en los estudiantes para despertar su interés por la investigación.

Los grandes cambios sociales ocurridos con el triunfo revolucionario de 1959 determinaron la transformación de la educación superior cubana comienza una época de perfeccionamiento educacional que llega hasta nuestros días. Los estudios universitarios en los primeros años mantienen los principios normativistas y positivistas de etapas anteriores a 1959. En la década de los 60 del pasado siglo las carreras universitarias se ven limitadas desde el Curriculum a priorizar la investigación como componente de formación.

Así durante más de 20 años se mantienen una formación academicista en detrimento de la investigativa en la Universidad cubana. A finales de la década del 80 se pone en vigor el Plan de estudios C y su posterior perfeccionamiento, El nuevo plan de estudios se caracterizó por (Fernández, 1997, p. XI):

- La conjugación armónica entre la teoría y la práctica.

- Incorporación del componente investigativo.

- Aplicación efectiva de los métodos activos de enseñanza.

- Enseñar a pensar y a investigar a los estudiantes de manera que construyan su propio camino del conocimiento.

- Sentar y recoger doctrina, como guía en la solución de los problemas jurídicos internos y como puente de la integración cultural americana.

\section{La formación de profesionales emprendedores: reto de la Universidad contemporánea}

En América Latina desde finales de la década del 80 hasta 2000 se han desarrollado distintas iniciativas para promover el emprendimiento y se ha establecido un esquema pedagógico y metodológico que permite la creación de empresas y el estímulo de la mentalidad empresarial.

Estudios actuales han demostrado que el tema del emprendimiento ya no es una exclusividad para el sector empresarial, sino que su importancia ha trascendido ha adquirido, y se ha llegado a convertir en una herramienta para la creación de empleo y generación de riqueza, lo cual demuestra que el crecimiento y el desarrollo económico están ligados al emprendimiento.

Para algunos autores como Tarapuez, Osorio y Botero (2013), el emprendimiento "es una manera de pensar y actuar orientada hacia la creación de riqueza, centrada en las oportunidades, planteada con visión global y llevada a cabo mediante un liderazgo equilibrado y la gestión de un riesgo calculado".

Otro referente importante lo encontramos en Ademar (2011), que define el emprendimiento como

La manera de resolver problemas con iniciativa y creatividad y desempeñar un papel más protagónico en la construcción de sus propios proyectos de vida, de modo que los estudiantes puedan actuar como verdaderos autores del cambio en los procesos sociales, políticos, culturales y económicos que les corresponde vivir, para que contribuyan al desarrollo sostenible de su comunidad (pp. 9-28).

Así en la actualidad la formación de profesionales emprendedores es una prioridad, y se necesitan respuestas desde las políticas universitarias, para combinar la investigación con el emprendimiento desde la teoría y desde la práctica. Así las Ciencias de la Educación, en particular la Pedagogía brinda una plataforma epistemológica que le permite la fundamentación de este importante proceso. 
Así se experimentan progresos en el tema del emprendimiento desde el punto de vista de la formación. Las universidades latinoamericanas han avanzado notablemente en la oferta de cursos de pregrado y posgrados. Es evidente cómo hoy éstas cuentan en sus estructuras con áreas de apoyo al emprendimiento. Su enseñanza en las universidades de la región no sólo es pertinente para los que estudian carreras de administración, economía y afines, sino para quienes se forman en otros campos, como las ingenierías.

Se destaca la figura del emprendedor como centro del ecosistema. Como el elemento más importante, los representantes de las diferentes instituciones (universidades, empresas, gobiernos) deben trabajar para ellos. Se debe entender que el proyecto y/o empresa es medio y resultado que demuestra su capacidad emprendedora. Por ende, es importante en esta dinamiza focalizar el papel del emprendedor, sus necesidades, potencialidades y limitacio- nes, así como los niveles de ayuda que necesita para superarse.

Otros aspectos importantes son las relaciones contextuales del emprendedor, sus competencias sociales, por lo que concientizarlo en la comprensión de la importancia de hacer relaciones que permitan construir confianza para facilitar el emprendimiento. Así, crear redes de inversionistas para los distintos tipos de emprendimiento, genera oportunidades de avanzar en este camino arduo y difícil, pero necesario.

Por todo lo anterior es importante el diseño de políticas institucionales para que desde las Universidades se promuevan el emprendimiento institucional, la generación de ideas y la cultura innovadora, encaminados a transformar el entorno de manera positiva. Esta política necesita de hacer articular las estrategias y acciones de las facultades, programas y las distintas áreas, que promuevan la cultura emprendedora en la comunidad, mediante líneas de trabajo, derivadas de los retos en términos de emprendimiento, innovación y sostenibilidad, manteniendo una alineación con las tendencias globales.

\section{METODOLOGÍA}

Para el siguiente trabajo de investigación se empleó una metodología basada en el paradigma cualitativo que asume como base metodológica la concepción dialéctica en tanto posibilitó: el estudio del objeto como un proceso y la fundamentación e integración de los otros métodos utilizados tales como:

Método teórico: el histórico-lógico, utilizado para la determinación de las principales características en el proceso de formación investigativa en el contexto contemporáneo, su particularidad en Cuba. Como métodos empíricos se utilizaron la encuesta a estudiantes y profesores y el análisis documental de los principales documentos metodológicos vigentes. El método estadístico (sistema SPS) permitió el procesamiento de los datos obtenidos en los instrumentos aplicados.

\section{RESULTADOS}

La presente investigación parte del reconocimiento empírico de que el proceso de formación investigativa en función del emprendimiento de los estudiantes de la Universidad de Pinar del Río presenta insuficiencias en su implementación, que se manifiestan en la no correspondencia entre los objetivos académicos de cada año y la formación de las habilidades de investigación, en la inexistencia de estrategias para formar competencias emprendedoras desde la docencia en sus diferentes modalidades, en un manejo inadecuado de las categorías de la ciencia y en el no reconocimiento de la importancia de la investigación científica.

Con el objetivo de analizar las manifestaciones del proceso que se estudió en los estudiantes de la facultad de Educación Media y Media Superior Universidad de Pinar del Río, se elaboraron varios instrumentos:

- Una encuesta de 6 preguntas para los estudiantes de 1ero, 2 do y 3 er años de la carrera.

- Una encuesta de 8 preguntas para los estudiantes de 4to año de la carrera.

- Una encuesta de 9 preguntas para los profesores de la UPR.

- Análisis documental de los principales documentos de las carreras de la facultad. 
De un total de 110 estudiantes, se encuestaron 103 para un 93.63\%. Por interés de nuestra investigación a los estudiantes de 4 año que fueron seleccionados para realizar trabajo de diploma (10 en total), se les aplicó un instrumento diferente, con el objetivo de constatar de forma más profunda como los conocimientos y habilidades adquiridas durante toda la carrera le podía ayudar en su trabajo de grado.

Los instrumentos aplicados arrojaron los siguientes resultados cuantitativos:

- El 62 \% de los estudiantes reconoce que la investigación no es importante en su formación como futuro profesional.

- Solo el $52.2 \%$ de los estudiantes reconoce, y solo en parte, la contribución de los contenidos al desarrollo de las investigaciones.

- La búsqueda bibliográfica (94.1\%) y la fundamentación de criterios (60 \%) son las acciones más frecuentes realizadas por los estudiantes para trabajos investigativos.

- El $77.6 \%$ de los estudiantes considera que las asignaturas desarrollan acciones que no exigen del desarrollo de la creatividad y la innovación.

- $\quad$ El 53,8 \% de los profesores encuestados tuvieron alguna dificultad para identificar de forma precisa los modos de actuación del profesional.

- El 93 \% de los profesores reconoce como necesario un proceso de formación de habilidades investigativas sistémico y contextualizado.

El estudio diagnóstico del proceso de formación investigativa en función del emprendi- miento de los estudiantes de la Universidad de Pinar del Río permitió determinar una serie de hallazgos:

- La actividad investigativa no es considerada importante para la correcta formación del profesional.

- Los contenidos de las asignaturas no favorecen acciones necesarias para lograr el desarrollo de la creatividad, la innovación y el emprendimiento.

- No se logra la necesaria unidad entre el componente teórico y el práctico en la realización de la investigación orientada al emprendimiento.

\section{CONCLUSIONES}

El proceso de formación investigativa en función del emprendimiento de los estudiantes de la Universidad de Pinar del Río debe estar dirigido al fomento de un espíritu innovador creativo como eje transversal para toda la planeación.

Integrar la formación desde lo académico, laboral, investigativo y extensionista permitirá formar competencias emprendedoras en los universitarios.

La labor del docente universitario, como gestor y modelo de actuación profesional, es esencial para el fomento de la investigación desde una postura de emprendimiento.

El análisis realizado sobre el proceso de formación de habilidades investigativas en el contexto actual, evidencia sus principales problemas y los retos a enfrentar por las instituciones de educación superior.

Los centros de educación superior deben convertirse en los principales gestores de profesionales competentes y capacitados para enfrentar y transformar su realidad social.

La investigación científica constituye el nexo natural y necesario entre los profesionales egresados de la educación superior y las necesidades de su contexto social.

Los estudios de derecho tienen como misión primordial fomentar una investigación que, fundamentada en un paradigma holístico, reconozca la necesaria relación entre la ciencia jurídica y las ciencias sociales. 


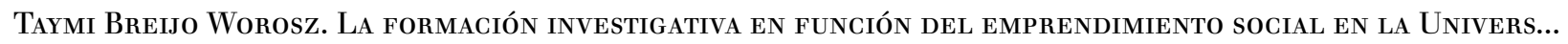

\section{ReFERENCIAS}

Ademar. (2011). Universidad Católica de Colombia. Lineamientos de emprendimiento institucional. Bogotá: Ucatolica.

Alfonso, P. (2002). El papel de la universidad en el proceso de formación para la economía social y el cooperativismo. Recuperado de http://www.sappiens.com/\%20Castellano/art\%C3\%ADculos.nsf/Cultural

Álvarez, C y Sierra, V. (1997). La Universidad. Sus procesos y su evaluación institucional. Pedagogía universitaria. Pinar del Río, Cuba: Universidad de Pinar del Río.

Fernández, J. (1997). Filosofía del Derecho. Editorial Félix Varela. La Habana.

Munárriz, S. (2008). La investigación universitaria ante los desafíos de la globalización. Recuperado de https:// es.scribd.com/document/202174605/La-Investigacion-Universitaria-Ante-Los-Desafios-de-La-Globalizacion

Tarapuez, Osorio y Botero (2013). Universidad Católica de Colombia. Lineamientos de emprendimiento institucional. Bogotá: Ucatolica.

Unesco. (1995). Documento de Política para el Cambio y el Desarrollo en la Educación Superior. París: Organización de las Naciones Unidas para la Educación, la Ciencia y la Cultura. Recuperado de https://unesdoc.unesco.org/ ark:/48223/pf0000098992_spa

Vitier, M. (2002). Las ideas en Cuba. La Filosofía en Cuba. Editorial Ciencias Sociales. La Habana. 